\title{
MiR-30c regulates metastasis and polarity reversal of tumor cell clusters by targeting MTDH in invasive micropapillary carcinoma of the breast
}

\section{Yunwei Han}

tianjin tumor hospital

\section{Weidong Li}

tianjin tumor hosptial

Renyong Zhi

tianjin tumor hosptital

Gui Ma

tianjin nankai university

Ang Gao

tianjin nankai university

Kailiang Wu

tianjin tumor hospital

Yawen Song

tianjin tumor hospital

Hui Sun

Tianjin tumor Hospital

Dan Zhao

tianjin nankai university

Yiling Yang

tianjin tunor hospital

Fangfang Liu

tianjin tunor hospital

Feng Gu

tianjin tumor hospital

\section{Xiaojing Guo}

tianjin tunor hospital

Jintang Dong

tianjin nankai university

Shuai Li

tianjin tumor hospital

Li Fu ( $\square$ fuli@tijmu.edu.cn ) 
Tianjin Tumor Hospital

\section{Research}

Keywords: Invasive micropapillary carcinoma (IMPC), miRNA, Polarity reversal, Invasion, Metastasis

Posted Date: September 8th, 2020

DOI: https://doi.org/10.21203/rs.3.rs-69356/v1

License: (c) (i) This work is licensed under a Creative Commons Attribution 4.0 International License. Read Full License 


\section{Abstract \\ Purpose}

Invasive micropapillary carcinoma (IMPC) of the breast has a high propensity for lymphovascular invasion and axillary lymph node metastasis and displays an 'inside-out' growth pattern, but the molecular mechanism of metastasis and tumor cell polarity reversal in IMPC is unclear.

\section{Methods and Patients:}

Luciferase reporter assays and western blotting were performed to confirm that the MTDH $3^{\prime} U T R$ bound to miR-30c. Growth curves, tumor sphere formation assays, Transwell migration and invasion assays, mouse xenograft model and immunofluorescence were evaluated to investigate the effects of miR-30c and MTDH. MiRNA in situ hybridization (ISH) and immunohistochemistry (IHC) were carried out on IMPC patient tissues for miR-30c and MTDH expression, respectively.

\section{Results}

We found that miR-30c could directly target the MTDH (metadherin) 3'UTR. MiR-30c overexpression inhibited breast cancer cell proliferation, invasion and metastasis by targeting MTDH in vitro and in vivo. Moreover, miR-30c regulated IMPC cell polarity reversal by targeting MTDH. By in situ hybridization and immunohistochemistry analyses, miR-30c and MTDH were significantly correlated with tumor size, lymph nodule status and tumor grade, the 'inside-out' growth pattern, overall survival (OS) and disease-free survival (DFS) in IMPC patients $(p<0.05)$.

\section{Conclusions}

Overall, miR-30c overexpression could inhibit breast cancer cell proliferation and metastasis and regulate polarity reversal by targeting MTDH.

\section{Introduction}

IMPC is an aggressive subtype of breast cancer (2003 WHO classification of breast cancer) characterized by small papillary structures lacking true central fibrovascular cores and special growth patterns of clusters of cells with reversed polarity [1, 2]. IMPC tumor cell clusters display an 'inside-out' growth pattern with an apical surface facing the basement membrane and a basal surface binding to the lumen. This micropapillary growth pattern has also been identified in multiple tumor tissues, such as gastric [3], colon [4], and lung [5] and is associated with poor prognosis. Although the incidence of IMPC ranges from 2 to $8 \%$ of all primary breast cancers, it exhibits more aggressive characteristics, such as lymphovascular 
invasion, lymph node metastasis, and distant metastasis [6-12]. Recently, precise pathologic diagnosis and individualized treatment have improved the outcomes of IMPC patients [13].

microRNA (miRNA), a kind of short noncoding RNA (ncRNA), plays vital regulatory roles in multiple biological processes, including cell proliferation, differentiation, autophagy, apoptosis and senescence $[14,15]$. More than half of human miRNA genes are localized within fragile genomic regions, which are subject to different structural genomic alterations during cancer development [16]. The dysregulation of miRNA expression appears to be a hallmark of metastatic breast cancer [17]. For example, miR-10b overexpression could positively regulate cell migration and invasion by targeting the prometastatic gene RHOC [18]. The high levels of serum miR-103 could target multiple endothelial junction genes and promote tumor metastasis [19]. Our previous study showed that miR-30c was significantly downregulated in IMPC compared with IDC-NST [20]. The potential role of miR-30c downregulation in IMPC needs to be further investigated.

In the current study, we found that miR-30c could inhibit breast cancer cell proliferation, invasion and metastasis by targeting MTDH both in vitro and in vivo. MiR-30c negatively regulated MTDH, which may be involved in modifying the apico-basal polarity of IMPC. We also revealed that miR-30c and MTDH were significantly associated with multiple clinical pathological characteristics, prognosis and the 'inside-out' growth pattern of IMPC patients. These findings suggest that the miR-30c/MTDH axis plays an important role in metastasis and the polarity reversal of tumor cell clusters and may serve as a prognostic marker of IMPC.

\section{Materials And Methods}

\section{Clinical tumor specimens}

Primary breast cancer specimens were obtained from the tissue bank at the Department of Breast Cancer Pathology and Research Laboratory, Tianjin Medical University Cancer Institute and Hospital, Tianjin, China. Formalin-fixed paraffin-embedded (FFPE) primary tumor specimens of IMPC ( $n=124$, from Jan 2007 to Dec 2012) and the period corresponding to IDC-NST $(n=149)$ were diagnosed by three independent pathologists. No patients included in this study received any chemotherapy or radiotherapy before the surgery, and their complete clinical data were available. The histologic type determination was based on the tumor-node-metastasis (TNM) staging system, reclassified according to the World Health Organization (WHO) classification. The date of death and the date of relapse were used to calculate overall survival (OS) and disease-free survival (DFS). OS was calculated from diagnosis to the date of death. DFS was defined as the time from diagnosis to disease progression or death, regardless of which occurred first. In IMPC patients, with a median follow-up of 72 months, recurrence or distant organ metastasis was observed in 33 patients, and 22 patients died. This study was approved by the Ethics Committee of Tianjin Medical University Cancer Institute and Hospital.

\section{Cell lines and culture}


Breast cancer cell lines (MCF-7, T47D, BT474, MDA-MB-231), HEK293 and HEK293T cells were used in this study. The cell lines were purchased from American Type Culture Collection (ATCC, Manassas, USA). Cells were cultured according to the manufacturer's protocols. As their phenotypic characteristics and patterns of genomic copy number variation (CNV) are consistent with those of the IMPC specimens [21], the ER-positive breast cancer cell lines MCF-7, T47D and BT474 were used in this study. All cells were cultured at $37^{\circ} \mathrm{C}$ and $5 \% \mathrm{CO}_{2}$.

\section{miRNA in situ hybridization and protein immunohistochemical analysis}

miRNA in situ hybridization (ISH) procedures were conducted as previously described [22]. Tumor sections were digested with proteinase $\mathrm{K}$ for $10 \mathrm{~min}$ and hybridized with hybridization solution at $54^{\circ} \mathrm{C}$ for $2 \mathrm{~h}$ with double-DIG-LNA probes for miR-30c, U6 (positive control) or scrambled RNA (negative control) (Exiqon, Denmark) (Supplementary Fig. S1). After washing with PBS, the slides were incubated with sheep anti-DIG-AP (Roche, Switzerland) and then stained with nitroblue tetrazolium/5-bromo-4- chloro-3indolyl-phosphate (Roche, Switzerland). Nuclear Fast Red was used to counterstain the nuclei. A positive signal appeared blue in the cytoplasm and nucleus. The intensities of miR-30c staining were scored as $0-4$, according to previously described standards [23].

Immunohistochemical staining was performed on FFPE tissue sections. The sections were incubated with primary antibodies against MTDH (Invitrogen 40-6500, USA), MUC1 (Abcam ab45167, England), Vimentin (Cell Signaling Technology 3932, USA), N-cadherin (BD Biosciences 610920, USA), E-cadherin (Cell Signaling Technology 3195, USA), and Ki67 (Thermo Fisher Scientific RM9106S0, USA) overnight at $4{ }^{\circ} \mathrm{C}$. Then, the sections were treated with biotinylated antibody, streptavidin-biotin-peroxidase and DAB complex (Beijing Zhongshan Biotechnology, China). The nuclei were counterstained with hematoxylin. The intensities of MUC1 and MTDH staining were scored between 0 and 4 as previously described [24, 25].

\section{Vector construction and lentivirus production}

MTDH 3'UTR fragments containing miR-30c putative targeting sites were subcloned into the firefly luciferase 3'UTR. Point mutations of miR-30c targeting sites were generated by a plasmid site-directed mutagenesis kit (Takara, Japan).

The pLKO.1-puro empty vector and plasmids containing shRNA against MTDH were purchased from Sigma. Lentiviral particles were produced in HEK293T cells by cotransfecting the cells with pLKO.1, pMD2.G and psPAX2 plasmids using Lipofectamine 2000 reagent (Invitrogen, CA) according to the manufacturer's protocol.

Cells were cultured in 24-well plates with a transfection complex. Approximately $5 \mu \mathrm{l}$ of $20 \mu \mathrm{M}$ chemically synthesized miR-30c mimic, miR-30c inhibitor or negative siRNA control was used. Ectopic expression of miR-30c, MTDH and MUC1 was achieved by infection with lentiviral solution (GenePharma, China) along with vector control lentiviral supernatant containing $5 \mu \mathrm{g} / \mathrm{ml}$ polybrene (Sigma, USA). After the cells reached $90 \%$ confluency, $2 \mu \mathrm{g} / \mathrm{ml}$ puromycin (Sigma, USA) was added to the medium for $3-5$ days to 
select cells with stable overexpression. Stable cells were maintained in medium containing $1 \mu \mathrm{g} / \mathrm{ml}$ puromycin.

\section{Western blots}

Cells were washed with PBS twice, and then proteins were extracted, separated by $10 \%$ SDS PAGE electrophoresis and transferred to PVDF membranes (Millipore, USA). To block nonspecific binding, the membranes were incubated with $5 \%$ skim milk for $1 \mathrm{~h}$ at room temperature. The membranes were then incubated overnight at $4{ }^{\circ} \mathrm{C}$ with an antiserum containing antibodies against MTDH (Invitrogen 40-6500, USA), MUC1 (Abcam ab45167, England), ß-actin (Sigma-Aldrich A2228, USA), and p-p65 (Abcam 3033, England). WesternBright ECL (Advansta, CA) was used following standard protocols, and blots were imaged with the ImageQuant LAS 4000 luminescent image analyzer (General Electric, CT).

\section{Dual luciferase reporter assay}

HEK293 cells seeded in 24-well plates were cotransfected with $500 \mathrm{ng}$ firefly luciferase reporter vector containing the MTDH 3'UTR (named psiCHECK2-MTDH-3'UTR wt) or MTDH 3'UTR mutant (named psiCHECK2-MTDH-3'UTR mut) and $100 \mathrm{nM}$ of negative control siRNA or miR-30c mimics in a final volume of $0.5 \mathrm{ml}$ using Lipofectamine 2000 (Invitrogen, USA). Firefly and Renilla luciferase activities were measured consecutively using dual luciferase assays (Promega, WI) $48 \mathrm{~h}$ after transfection. All transfection experiments were performed in triplicate and reproduced at least three times.

\section{SRB assay and colony formation assay}

Transfected cells were plated into 24-well plates at $5 \times 10^{4}$ cells/well and cultured for different times. Cells were then fixed and stained with sulforhodamine B (SRB). For the colony formation assay, transfected cells were seeded in 6-well plates at 5000 cells per well. Fourteen days later, colonies were fixed with $4 \%$ paraformaldehyde and stained with crystal violet for $15 \mathrm{~min}$.

\section{Cell invasion and migration assay}

The migration assay was performed using a Transwell system (24-well, $8 \mu \mathrm{m}$ pore size with polycarbonate membrane; Corning Costar, USA) according to the manufacturer's protocol. The invasion assay was performed in the same way as the migration assay except the polycarbonate membranes were coated with $50 \mu \mathrm{l}$ growth factor-reduced Matrigel (BD Biosciences, USA) at $37^{\circ} \mathrm{C}$ for $1 \mathrm{~h}$ to form a reconstituted basement membrane. Then, $800 \mu$ l serum-containing ( $20 \%$ FBS) medium in each well was added to the lower well of each chamber. A total of $1 \times 10^{5}$ resuspended cells were added to the upper inserts with $100 \mu \mathrm{l}$ serum-free medium. After $48 \mathrm{~h}$, the remaining cells on the upper side of the Transwell device were removed, and the invading cells at the bottom of the Transwell were fixed with $4 \%$ paraformaldehyde and stained with crystal violet.

For wound healing assays, transfected cells were grown in complete medium, and $10 \mu$ pipette tips were used to create an artificial wound in 24-well plates. Cells were washed with PBS to remove the debris and were cultured for $24 \mathrm{~h}$ to allow wound healing. 


\section{Mouse xenograft model}

The animal experiment was approved by the Animal Care and Use Center of Nankai University, Tianjin, China. $17 \beta$-Estradiol pellets ( $0.72 \mathrm{mg}$, 90-day release; Innovative Research of America, $\mathrm{FL}$ ) were implanted subcutaneously in the shoulder region of each 4-week-old female BALB/c nude mouse (Charles River, China) one day before cell inoculation. MCF-7 cells $\left(1 \times 10^{7}\right)$ transfected with lenti-miR-30c or lenti-empty were resuspended in a solution of Matrigel (BD Biosciences, USA) or PBS (equal volume), respectively, and then inoculated subcutaneously into the flanks of BALB/c nude mice $(n=12)$. Tumor volumes were measured once a week and calculated as follows: $A \times B^{2} / 2$, where $A$ is the largest diameter and $B$ is the diameter perpendicular to $A$. Mice were sacrificed eight weeks after injection, and tumors were surgically removed, weighed, and fixed in $4 \%$ formalin. H\&E staining was performed for histological examination.

To evaluate the effect of miR-30c on tumor metastasis, $1 \times 10^{6}$ MDA-MB-231 cells infected with lenti-miR$30 \mathrm{c}$ or lenti-empty were injected into the tail veins of four-week-old female BALB/c nude mice (seven mice for each group). Four weeks later, the numbers of micrometastases in the lung per H\&E stained section from individual mice were analyzed by morphological observation.

\section{Three-dimensional (3D) morphogenesis assays}

A three-dimensional (3D) morphogenesis assay was performed as previously described (26). Eight-well chamber slides were loaded with $50 \mu$ of growth factor-reduced Matrigel (BD Biosciences, CA) and allowed to set for $1 \mathrm{~h}$. A total of 5000 treated cells/well were then overlaid onto the gel in DMEM supplemented with $10 \%$ FBS and $2 \%$ Matrigel and cultured for two weeks for counting using an inverted microscope.

\section{Statistics}

Statistical analyses were performed using GraphPad Prism 5 software (GraphPad, CA) and the SPSS 21.0 software package (SPSS Inc, USA). Clinicopathological variables between the IMPC and IDC-NST groups were analyzed using the Chi-square test. Correlations between miR-30c, MTDH or MUC1 expression and clinicopathological variables were evaluated by Mann-Whitney U-test and Spearman's rank correlation analysis. OS and DFS rates were estimated by Kaplan-Meier analysis. Cox proportional hazard regression analysis was performed for the identification of relevant prognostic factors. For tumorigenesis experiments, tumor sizes or weights between two groups were analyzed using Student's $t$ test. The statistical analysis was two-sided, and $P$-values less than 0.05 were considered statistically significant.

\section{Results}

\section{MiR-30c inhibits MTDH expression by targeting its 3'UTR}


Our previous research revealed that miR-30c was significantly downregulated in IMPC compared with IDC-NST [20]. To explore the downstream targets of miR-30c, we employed TargetScan, PicTar and DIANA bioinformatic tools to predict the target genes of miR-30c. As shown in Fig. 1a and b, MTDH was predicted as a miR-30c target by the three different algorithms. Moreover, the two predicted target sites were conserved among vertebrates (Fig. 1c). Two MTDH 3'UTR fragments containing the putative target sites were subcloned into the 3'UTR of the luciferase expression vector. As shown in Fig. 1d, miR-30c overexpression significantly downregulated luciferase activity in both reporter assays. Point mutations that interrupt base pairing between the miR-30c seed sequence and MTDH 3'UTR could block the inhibitory effect of miR-30c. We examined the expression of miR-30c and MTDH in a series of breast cancer cell lines (Supplementary Fig. 2A, B). Quantitative real-time PCR demonstrated that miR-30c mimics could efficiently overexpress miR-30c by transfection in MCF-7, T47D and MDA-MB-231 cells (Supplementary Fig. 2C). MiR-30c overexpression could downregulate the protein levels of MTDH while slightly inhibiting MTDH mRNA levels (Fig. 1e, f). This is consistent with the common view that mammalian miRNAs mainly regulate gene expression through translational inhibition. Moreover, antimiR-30c significantly increased the protein levels of MTDH (Supplementary Fig. 2D). All these results demonstrated that miR-30c regulated MTDH through direct binding to its $3^{\prime} U T R$.

\section{MiR-30c inhibits breast cancer cell proliferation in vitro by targeting MTDH}

As their phenotypic characteristics and genomic copy number variation (CNV) patterns are consistent with those of IMPCs [21], the breast cancer cell lines MCF-7, T47D and BT474 were used to mimic IMPC. We found that miR-30c overexpression significantly inhibited the proliferation and clonogenic ability of MCF-7, T47D and BT474 cells (Fig. 2a-f). MTDH overexpression partially abrogated the miR-30c-mediated suppression of breast cancer cell proliferation and protein expression (Fig. 2a-C, g, h). As shown in Fig. 2iI, miR-30c overexpression or MTDH knockdown significantly inhibited tumorsphere formation in breast cancer cells. To further confirm that miR-30c specifically regulates MTDH, we used anti-miR-30c to abrogate miR-30c expression in MDA-MB-231 and T47D cells. As expected, anti-miR-30c significantly induced MTDH expression with a concomitant increase in cell proliferation (Supplementary Fig. 2D-F). All these observations demonstrated that MTDH was a key mediator of the miR-30c-regulated signaling pathway in proliferation.

\section{miR-30c inhibits breast cancer cell migration and invasion in vitro by targeting MTDH}

Transwell migration and invasion assays and wound healing assays were performed on MCF-7, T47D and BT474 cells lines. As shown in Fig. 3a-h, miR-30c overexpression significantly suppressed breast cancer cell migration and invasion. Ectopic expression of the MTDH gene could promote cell migration and invasion. To further determine whether miR-30c exerts its function by inhibiting MTDH, we investigated the effects of miR-30c when MTDH was overexpressed. As expected, MTDH overexpression could rescue the inhibitory effects of miR-30c overexpression on cell migration and invasion (Fig. 3ah).Conversely, anti-miR-30c significantly induced MTDH expression with a concomitant increase in cell 
invasion and migration (Supplementary Fig. 3G, H). All these observations demonstrated that MTDH was a key mediator of the miR-30c-regulated signaling pathway in migration and invasion.

\section{miR-30c suppresses tumorigenesis and metastasis in a xenograft model}

To further explore the role of miR-30c in tumorigenesis in vivo, $1 \times 10^{7}$ MCF-7 cells infected with lenti-miR$30 \mathrm{c}$ or lenti-empty were inoculated subcutaneously into the flanks of twelve mice. Tumor volume was monitored every week, and tumor growth curves were plotted. Lentivirus-mediated miR-30c overexpression significantly inhibited tumor formation compared with that in the lenti-empty group (Fig. 4a-c). As indicated by Ki67 immunostaining, lenti-miR-30c significantly inhibited cell proliferation compared with that in the lenti-empty group in vivo (Fig. 4d).

To determine whether miR-30c overexpression is capable of blocking breast cancer cell lung metastases, MDA-MB-231 cells were infected with lenti-miRNA-30c or lenti-empty and then tail vein injected. Four weeks after injection, mice were sacrificed, and lung metastasis was investigated. As shown in Fig. 4F and $G$, pulmonary metastatic nodules were reduced in the lenti-miR-30c group compared with the lentiempty group. According to these results, enhanced expression of miR-30c decreased tumorigenesis and metastasis in vivo. In addition, immunohistochemistry and western blot analyses revealed that MTDH and E-cadherin expression levels were downregulated and $\mathrm{N}$-cadherin expression levels were upregulated in the lenti-miR-30c group compared with the lenti-empty group, indicating that miR-30c overexpression might inhibit the process of EMT (Fig. 4h, Supplementary Fig. 3A).

\section{miR-30c regulates the polarity reversal of tumor cell clusters in IMPC by targeting MTDH}

Mucin glycoproteins promote invasion, proliferation, and migration and regulate cell polarity through diverse pathways in epithelial cancers [27]. Since the morphological characterization of IMPC can be assessed by the 'inside-out' staining pattern of epithelial membrane antigen (MUC1), we hypothesized that the miR-30c/MTDH axis might regulate the 'inside-out' growth pattern of IMPC. Quantitative real-time PCR and western blot demonstrated that miR-30c mimics could overexpress miR-30c efficiently and downregulated the protein levels of MTDH in IMPC primary cells (Supplementary Fig. 3B, C). To further analyze the role of the miR-30c/MTDH axis in cell polarity, IMPC primary cells were used for polarity studies in vitro. The results showed that miR-30c overexpression could decrease the 'inside-out' staining pattern of MUC1, and LV6-MTDH overexpression could increase the 'inside-out' staining pattern of MUC1. MTDH overexpression rescued the inhibitory effects of miR-30c overexpression on the inside-out staining pattern of MUC1, as demonstrated by multidimensional invasive spherical mass analysis (Fig. 5a-d). Our results indicate that miR-30c negatively regulates the polarity reversal of tumor cell clusters in IMPC by targeting MTDH, but there is not a strong overlapping localization between MTDH and MUC1 observed in confocal images of immunofluorescence in IMPC primary cells (Supplementary Fig. 3D). 
Moreover, immunohistochemistry analysis revealed that MUC1 was downregulated in the lenti-miR-30c group compared with the lenti-empty group in the xenograft model (Fig. 4h, Supplementary Fig. 3A). Therefore, MUC1 may function downstream of miR-30c. MiR-30c overexpression suppressed phosphop65 (p-p65) and MUC1 expression in breast cancer cells (Fig. 5e). Since MTDH and MUC1 are involved in the NF-KB pathway [28, 29], we demonstrated that MTDH knockdown could downregulate phospho-p65 and MUC1 protein expression in MCF-7 and T47D cells (Fig. 5f). Therefore, the miR-30c/MTDH axis might regulate MUC1 through the NF-KB pathway. It has also been reported that MTDH regulates $\beta$-Catenin signaling to maintain glioma stem-like stemness and self-renewal [30]. However, according to our results, the $\beta$-Catenin signaling pathway might not be activated in IMPC.

\section{MiR-30c/MTDH is correlated with the clinicopathologic characteristics and prognosis of IMPC}

Consistent with our previous study [9], 86\% of IMPC patients and $45 \%$ of IDC-NST patients had lymph node metastasis, indicating a high lymph node metastasis rate in IMPC compared to IDC-NST. The prevalence of ER expression was $72 \%$ and $60 \%$ in IMPC and IDC-NST, respectively, comparable with previous reports by Chen AC and Tresserra F et al.[31, 32].

To explore miR-30c and MTDH expression in IMPC, we measured miR-30c and MTDH levels in 124 IMPC tissues and corresponding 149 IDC-NST tissues by ISH and IHC. Representative images of miR-30c, MTDH and MUC1 expression in IMPC and IDC-NST are shown in Fig. $6 a$ and b, demonstrating that miR$30 \mathrm{c}$ expression was remarkably downregulated in IMPC tissues compared with IDC-NST tissues ( 0.78 vs $0.62, p<0.05$ ) (Supplementary Table 1). Furthermore, we compared the expression levels of miR-30c between IMPC and IDC-NST paired tissues by quantitative real-time PCR $(n=11)$. The results showed that the expression of miR-30c was downregulated in IMPC tissues compared with IDC-NST tissues (Student's t-test, $p<0.05$, Fig. $6 \mathrm{~g}$ ). MTDH expression was significantly upregulated in IMPC tissues compared with IDC-NST tissues $(0.64$ vs $0.51, p<0.05)$ (Supplementary Table 1$)$. Low expression levels of miR-30c were correlated with larger tumor size, multiple lymph node metastases, higher tumor grade and higher histologic grade $(p=0.042, p<0.01, p<0.01$ and $p=0.013$, respectively; Supplementary Table 3$)$. The MTDH levels were significantly higher in IMPC tissues, and the high expression of MTDH was correlated with large tumor size, multiple lymph node metastases and higher tumor grade $(p=0.038, p<0.01$ and $p$ $<0.01$, respectively; Supplementary Table 3 ). To further study the association between miR-30c and MTDH expression, correlation analysis revealed that miR-30c was negatively correlated with MTDH in IMPCs (Supplementary Table 2). The polarity reversal phenotype of MUC1 was negatively correlated with the miR-30c level and positively correlated with the MTDH expression level in IMPC patients (Supplementary Table 2). These data indicated that miR-30c reduction might contribute to the overexpression of MTDH and lead to the polarity reversal of tumor cell clusters in IMPC.

Survival analysis showed that IMPC patients with high miR-30c expression had better OS and DFS than patients with low miR-30c expression ( $p=0.015$ and $p<0.01$, respectively; Fig. $6 c, \mathrm{e}$ ). OS and DFS were better in IMPC patients with low MTDH expression than high MTDH expression $(p<0.01$ and $p<0.01$, 
respectively; Fig. 6d, f). Univariate and multivariate survival analyses for OS and DFS were also performed. Tumor stage $(p=0.043)$ and $\operatorname{MTDH}(p=0.028)$ expression were independent risk factors for OS in IMPC (Supplementary Table 4). Nodal stage $(p=0.026)$ and MTDH $(p=0.01)$ expression were independent risk factors for DFS in IMPC (Supplementary Table 5). The results implicated a potential relationship between miR-30c and MTDH in the metastasis and prognosis of IMPC patients. Altogether, our results indicated that the miR-30c/MTDH axis might be important in the aggressive characteristics of IMPC (Fig. 6h).

\section{Discussion}

In the present study, for the first time, we demonstrated the important regulatory role of miR-30c in metastasis and polarity reversal of tumor cell clusters in IMPC. We found that miR-30c could negatively regulate MTDH expression by directly binding to its $3^{\prime} U T R$. MiR-30c overexpression inhibited cell proliferation, invasion and metastasis both in vitro and in vivo. MiR-30c regulated the polarity reversal of tumor cell clusters in IMPC cells by targeting MTDH. Moreover, we revealed that miR-30c and MTDH were significantly associated with multiple clinical pathological characteristics, prognosis, and the 'inside-out' growth pattern of IMPC.

IMPC is an aggressive subtype of breast cancer characterized by a special growth pattern of tumor cell polarity reversal, invasion and metastasis $[33,34]$. Previous research has demonstrated that miR-30c can regulate multiple tumorigenesis processes by targeting variable genes [35-37]. It is of great interest to explore the underlying mechanisms of miR-30c dysregulation in IMPC. Marchi $\mathrm{C}$ et al. showed that the genomic locations of miR-30c-1 and miR-30c-2, 1p34.2 and 6q13, respectively, were lost in IMPC [38]. The loss of chromatin regions may be responsible for the low expression of miR-30c in IMPC.

The dysregulation of MTDH is associated with several cancer-related processes, including proliferation, cell death, invasion, endothelial adhesion, metastasis and angiogenesis [38-41]. Hu et al. found that the MTDH genomic region 8q22 was frequently amplified in breast cancer, which led to MTDH overexpression, thereby promoting chemoresistance and metastasis of breast cancer patients with poor prognosis [39]. Genomic DNA deep sequencing results revealed that the entire 8q region was amplified in breast IMPC [42]. In the present study, by reporter assay and western blotting, we showed that miR-30c could target MTDH. Zhang et al. found that miR-30a could suppress proliferation, invasion and metastasis by targeting MTDH [41]. However, our previous real-time PCR results showed that only miR30c (not miR-30a) was downregulated in IMPC compared with IDC-NST [20]. Using cell culture and xenograft models, the current research revealed that dysregulated miR-30c might posttranscriptionally contribute to the overexpression of MTDH, thereby enhancing the aggressive characteristics of IMPC.

Loss of apico-basal polarity is increasingly being recognized as an important factor in tumor treatment [43]. Several IMPC-specific genes and ontology pathways are involved in the regulation of cell polarity, adhesion and migration [44]. Accordingly, elucidation of the molecular mechanism of polarity reversion in IMPC may contribute to understanding the metastasis mechanism of breast cancer. MUC1 is localized to 
the external surface of the IMPC cell clusters, and this particular architecture has been described as an 'inside-out' growth pattern [33]. The MUC1 staining pattern that evolves beyond apical localization might be associated with a more malignant phenotype [45]. There is evidence that MUC1 is involved in the initial attachment of carcinoma cells to tissues at distant sites, which may facilitate the establishment of metastatic foci [46]. Our previous findings suggested that Sialyl Lewis X, an MUC1 epitope, might play an important role in lymph node metastasis in IMPC [34]. MTDH is also recruited during the maturation of the tight junction proteins ZO-1 and occludin in polarized epithelial cells [47], suggesting that MTDH is involved in cell polarity regulation. Overall, our study indicated that miR-30c negatively regulated MTDH, resulting in tumor cell cluster polarity reversal and might contribute to invasion and metastasis of IMPC.

In conclusion, we identified a novel miRNA-mediated pathway that regulated metastasis and polarity reversal of tumor cell clusters in IMPC. The components of this pathway, miR-30c, MTDH, and MUC1, represent attractive targets for developing new therapeutic strategies in the management of breast cancer, especially in IMPC.

\section{Conclusion}

Invasive micropapillary carcinoma (IMPC) of the breast has a high propensity for lymphovascular invasion and axillary lymph node metastasis and displays an 'inside-out' growth pattern, but the molecular mechanism of metastasis and polarity reversal of tumor cell clusters in IMPC is unclear. Here, by bioinformatic prediction, reporter assay and western blotting, we found that miR-30c could directly target the MTDH (metadherin) 3'UTR. MiR-30c overexpression inhibited breast cancer cell proliferation, invasion and metastasis by targeting MTDH in vitro and in vivo. In a three-dimensional culture model, miR-30c negatively regulated MTDH and thereby led to cell polarity reversal. Moreover, by in situ hybridization analysis, we demonstrated that miR-30c and MTDH significantly correlated with clinical and pathological characteristics, prognosis, and the 'inside-out' growth pattern in IMPC patients. Overall, for the first time, we revealed that the miR-30c/MTDH axis resulted in metastasis and polarity reversal in IMPC. The miR-30c/MTDH axis might serve as a prognostic marker of IMPC.

\section{Abbreviations}

IMPC: Invasive micropapillary carcinoma; IDC-NST:Invasive ductal carcinoma no special type; ISH:in-situ hybridization; IHC Immunohistochemistry; ER:Estrogen receptor; PR:Progestrone receptor; HER2:Human epidermal growth factor receptor 2; OS:Overall survival; DFS:Disease-free survival.

\section{Declarations}

\section{Acknowledgements}

We thank Prof. Zhengmao Zhu, Ms. Liya Fu, Mr. Xing Fu Mr. Jun A, Mr. Mingcheng Liu, Ms. Qingxia Hu, Ms.Changying Fu, Ms. Ge Dong and Ms. Qiuying Li of Nankai University for helpful comments and 
assistance during the study.

\section{Abbreviations}

IMPC: Invasive micropapillary carcinoma; IDC-NST: Invasive ductal carcinoma no special type; ISH: in-situ hybridization; IHC Immunohistochemistry; ER: Estrogen receptor; PR: Progestrone receptor; HER2: Human epidermal growth factor receptor 2; OS: Overall survival; DFS: Disease-free survival.

\section{The authors declare no competing financial interests}

\section{Financial Support}

This study was funded by National Natural Science Foundation of China (81672637, 81872164), Cancer Translational Medicine Seed Fund of Tianjin Medical University Cancer Institute and Hospital (1804).

\section{Availability of data and materials}

This data that supports the findings of this manuscript can be made available from the corresponding author following reasonable request

\section{Ethics approval and consent to participate}

The studies were approved by an independent ethics committee. All patients provided written informed consent before study enrollment.

\section{Consent for publication}

Not applicable.

\section{Author contributions statement}

YWH, SL and LF conceived the project; YWH,WDL, RYZ, KLW, GM, AG, DZ, SL and LF designed the experiments and analyzed the data; YWH, GM, AG, RYZ, KLW,YWS, HQJ, QQS, BBL, performed the experiment; YLY, FFL, XJG contributed to the acquisition and analysis of data; YWH and SL prepared the manuscript; FG, JTD, SL and LF contributed to the critical review of the manuscript and the financial support of the work. All the authors have reviewed the manuscript and approved the final version.

\section{References}

1. Siriaunkgul S, Tavassoli FA. Invasive micropapillary carcinoma of the breast. Mod Pathol. 1993;6(6):660-2.

2. Bocker W. [WHO classification of breast tumors and tumors of the female genital organs: pathology and genetics]. Verh Dtsch Ges Pathol. 2002;86:116-9. 
3. Ohtsuki Y, Kuroda N, Yunoki S, Murakami S, Mizukami Y, Okada Y, et al. Immunohistochemical analysis of invasive micropapillary carcinoma pattern in four cases of gastric cancer. Med Mol Morphol. 2013;46(2):114-21.

4. Verdu M, Roman R, Calvo M, Rodon N, Garcia B, Gonzalez M, et al. Clinicopathological and molecular characterization of colorectal micropapillary carcinoma. Mod Pathol. 2011;24(5):729-38.

5. Koga K, Hamasaki M, Kato F, Aoki M, Hayashi H, Iwasaki A, et al. Association of c-Met phosphorylation with micropapillary pattern and small cluster invasion in pT1-size lung adenocarcinoma. Lung Cancer. 2013;82(3):413-9.

6. Paterakos M, Watkin WG, Edgerton SM, Moore DH 2nd, and Thor AD. Invasive micropapillary carcinoma of the breast: a prognostic study. Hum Pathol. 1999;30(12):1459-63.

7. Walsh MM, Bleiweiss IJ. Invasive micropapillary carcinoma of the breast: eighty cases of an underrecognized entity. Hum Pathol. 2001;32(6):583-9.

8. Guo X, Fan Y, Lang R, Gu F, Chen L, Cui L, et al. Tumor infiltrating lymphocytes differ in invasive micropapillary carcinoma and medullary carcinoma of breast. Mod Pathol. 2008;21(9):1101-7.

9. Chen L, Fan Y, Lang RG, Guo XJ, Sun YL, Cui LF, et al. Breast carcinoma with micropapillary features: clinicopathologic study and long-term follow-up of 100 cases. Int J Surg Pathol. 2008;16(2):155-63.

10. Fu L, Ikuo M, Fu XY, Liu TH, Shinichi T. [Relationship between biologic behavior and morphologic features of invasive micropapillary carcinoma of the breast]. Zhonghua Bing Li Xue Za Zhi. 2004;33(1):21-5.

11. Liu F, Yang M, Li Z, Guo X, Lin Y, Lang R, et al. Invasive micropapillary mucinous carcinoma of the breast is associated with poor prognosis. Breast Cancer Res Treat. 2015;151(2):443-51.

12. Guo X, Chen L, Lang R, Fan Y, Zhang X, Fu L. Invasive Micropapillary Carcinoma of the Breast. Am J Clin Pathol. 2006;126(5):740-46.

13. Li W, Han Y, Wang C, Guo X, Shen B, Liu F, et al., Precise pathologic diagnosis and individualized treatment improve the outcomes of invasive micropapillary carcinoma of the breast: a 12-year prospective clinical study. Mod Pathol. 2018.

14. Yu Z, Baserga R, Chen L, Wang C, Lisanti MP, Pestell RG. microRNA, cell cycle, and human breast cancer. Am J Pathol. 2010;176(3):1058-64.

15. Kong YW, Ferland-McCollough D, Jackson TJ, Bushell M. microRNAs in cancer management. Lancet Oncol. 2012;13(6):e249-58.

16. Calin GA, Sevignani C, Dumitru CD, Hyslop T, Noch E, Yendamuri S, et al. Human microRNA genes are frequently located at fragile sites and genomic regions involved in cancers. Proc Natl Acad Sci U S A. 2004;101(9):2999-3004.

17. Wang L, Wang J. MicroRNA-mediated breast cancer metastasis: from primary site to distant organs. Oncogene. 2012;31(20):2499-511.

18. Ma L, Teruya-Feldstein J, Weinberg RA. Tumour invasion and metastasis initiated by microRNA-10b in breast cancer. Nature. 2007;449(7163):682-8. 
19. Fang JH, Zhang ZJ, Shang LR, Luo YW, Lin Y, Yuan Y, et al., Hepatoma cell-secreted exosomal microRNA-103 increases vascular permeability and promotes metastasis by targeting junction proteins. Hepatology. 2018.

20. Li S, Yang C, Zhai L, Zhang W, Yu J, Gu F, et al. Deep sequencing reveals small RNA characterization of invasive micropapillary carcinomas of the breast. Breast Cancer Res Treat. 2012;136(1):77-87.

21. Natrajan R, Wilkerson PM, Marchio C, Piscuoglio S, Ng CK, Wai P, et al. Characterization of the genomic features and expressed fusion genes in micropapillary carcinomas of the breast. J Pathol. 2014;232(5):553-65.

22. Deng M, Tang HL, Lu XH, Liu MY, Lu XM, Gu YX, et al. miR-26a suppresses tumor growth and metastasis by targeting FGF9 in gastric cancer. PLoS One. 2013;8(8):e72662.

23. Tang H, Liu P, Yang L, Xie X, Ye F, Wu M, et al. miR-185 suppresses tumor proliferation by directly targeting E2F6 and DNMT1 and indirectly upregulating BRCA1 in triple-negative breast cancer. Mol Cancer Ther. 2014;13(12):3185-97.

24. Li J, Zhang N, Song LB, Liao WT, Jiang LL, Gong LY, et al. Astrocyte elevated gene-1 is a novel prognostic marker for breast cancer progression and overall patient survival. Clin Cancer Res. 2008;14(11):3319-26.

25. Sinn BV, von Minckwitz G, Denkert C, Eidtmann H, Darb-Esfahani S, Tesch H, et al. Evaluation of Mucin-1 protein and mRNA expression as prognostic and predictive markers after neoadjuvant chemotherapy for breast cancer. Ann Oncol. 2013;24(9):2316-24.

26. Horwitz KB, Dye WW, Harrell JC, Kabos P, Sartorius CA. Rare steroid receptor-negative basal-like tumorigenic cells in luminal subtype human breast cancer xenografts. Proc Natl Acad Sci U S A. 2008;105(15):5774-9.

27. Reynolds IS, Fichtner M, McNamara DA, Kay EW, Prehn JHM, Burke JP. Mucin glycoproteins block apoptosis; promote invasion, proliferation, and migration; and cause chemoresistance through diverse pathways in epithelial cancers. Cancer Metastasis Rev. 2019.

28. Sarkar D, Park ES, Emdad L, Lee SG, Su ZZ, Fisher PB. Molecular basis of nuclear factor-kappaB activation by astrocyte elevated gene-1. Cancer Res. 2008;68(5):1478-84.

29. Ahmad R, Raina D, Joshi MD, Kawano T, Ren J, Kharbanda S, et al. MUC1-C oncoprotein functions as a direct activator of the nuclear factor-kappaB p65 transcription factor. Cancer Res. 2009;69(17):7013-21.

30. Hu B, Emdad L, Kegelman TP, Shen XN, Das SK, Sarkar D, et al. Astrocyte Elevated Gene-1 Regulates beta-Catenin Signaling to Maintain Glioma Stem-like Stemness and Self-Renewal. Mol Cancer Res. 2017;15(2):225-33.

31. Chen AC, Paulino AC, Schwartz MR, Rodriguez AA, Bass BL, Chang JC, et al. Population-based comparison of prognostic factors in invasive micropapillary and invasive ductal carcinoma of the breast. Br J Cancer. 2014;111(3):619-22.

32. Tresserra F, Grases PJ, Fabregas R, Fernandez-Cid A, Dexeus S. Invasive micropapillary carcinoma. Distinct features of a poorly recognized variant of breast carcinoma. Eur J Gynaecol Oncol. 
1999;20(3):205-8.

33. Yang YL, Liu BB, Zhang X, Fu L. Invasive Micropapillary Carcinoma of the Breast: An Update. Arch Pathol Lab Med. 2016;140(8):799-805.

34. Wei J, Cui L, Liu F, Fan Y, Lang R, Gu F, et al. E-selectin and Sialyl Lewis X expression is associated with lymph node metastasis of invasive micropapillary carcinoma of the breast. Int J Surg Pathol. 2010;18(3):193-200.

35. Suh SS, Yoo JY, Cui R, Kaur B, Huebner K, Lee TK, et al. FHIT suppresses epithelial-mesenchymal transition (EMT) and metastasis in lung cancer through modulation of microRNAs. PLoS Genet. 2014;10(10):e1004652.

36. Fang $Y$, Shen $H$, Cao Y, Li H, Qin R, Chen Q, et al. Involvement of miR-30c in resistance to doxorubicin by regulating YWHAZ in breast cancer cells. Braz J Med Biol Res. 2014;47(1):60-9.

37. Bockhorn J, Dalton R, Nwachukwu C, Huang S, Prat A, Yee K, et al. MicroRNA-30c inhibits human breast tumour chemotherapy resistance by regulating TWF1 and IL-11. Nat Commun. 2013;4:1393.

38. Brown DM, Ruoslahti E. Metadherin, a cell surface protein in breast tumors that mediates lung metastasis. Cancer Cell. 2004;5(4):365-74.

39. Hu G, Chong RA, Yang Q, Wei Y, Blanco MA, Li F, et al. MTDH activation by 8q22 genomic gain promotes chemoresistance and metastasis of poor-prognosis breast cancer. Cancer Cell. 2009;15(1):9-20.

40. Wan L, Lu X, Yuan S, Wei Y, Guo F, Shen M, et al. MTDH-SND1 interaction is crucial for expansion and activity of tumor-initiating cells in diverse oncogene- and carcinogen-induced mammary tumors. Cancer Cell. 2014;26(1):92-105.

41. Zhang N, Wang X, Huo Q, Sun M, Cai C, Liu Z, et al. MicroRNA-30a suppresses breast tumor growth and metastasis by targeting metadherin. Oncogene. 2014;33(24):3119-28.

42. Marchio C, Iravani M, Natrajan R, Lambros MB, Savage K, Tamber N, et al. Genomic and immunophenotypical characterization of pure micropapillary carcinomas of the breast. J Pathol. 2008;215(4):398-410.

43. Ellenbroek SI, Iden S, Collard JG. Cell polarity proteins and cancer. Semin Cancer Biol. 2012;22(3):208-15.

44. Gruel N, Fuhrmann L, Lodillinsky C, Benhamo V, Mariani O, Cedenot A, et al. LIN7A is a major determinant of cell-polarity defects in breast carcinomas. Breast Cancer Res. 2016;18(1):23.

45. Siroy A, Abdul-Karim FW, Miedler J, Fong N, Fu P, Gilmore H, et al. MUC1 is expressed at high frequency in early-stage basal-like triple-negative breast cancer. Hum Pathol. 2013;44(10):2159-66.

46. Ciborowski P, Finn OJ. Non-glycosylated tandem repeats of MUC1 facilitate attachment of breast tumor cells to normal human lung tissue and immobilized extracellular matrix proteins (ECM) in vitro: potential role in metastasis. Clin Exp Metastasis. 2002;19(4):339-45.

47. Britt DE, Yang DF, Yang DQ, Flanagan D, Callanan H, Lim YP, et al. Identification of a novel protein, LYRIC, localized to tight junctions of polarized epithelial cells. Exp Cell Res. 2004;300(1):134-48. 
Figures

A

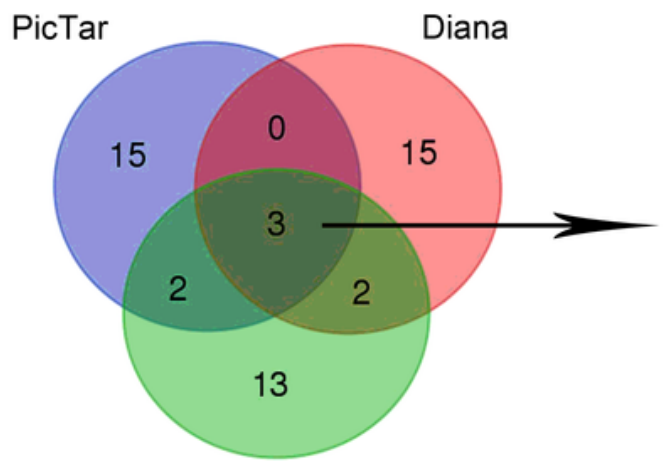

TargetScan

C

$287-294$

$1569-1576$

Human 5 '...GUGCCCUGUUUACAACAGA...GGCAGUAUGUUUACAUG...3' Chimp 5'...GUGCCCUGUUUACAACAGA...GGCAGUAUGUUUACAUG.... $3^{\prime}$ Rhesus 5 '...GUGCCCUGUUUACAACAGA...GGCAGUAUGUUUACAUG...3' Squirrel 5'...GUUCCCUGUUUACAACAGA...GGCAGUAUGUUUACAUG....3' Mouse $5^{\prime} \ldots$...GUUACCUGUUUACAACAGA...GGCAGUAUGUUUACAUG...3

$E$

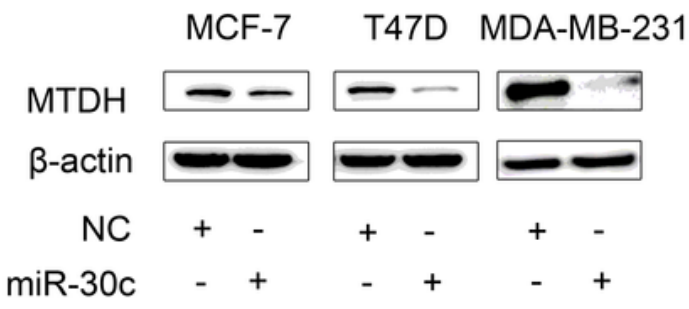

B

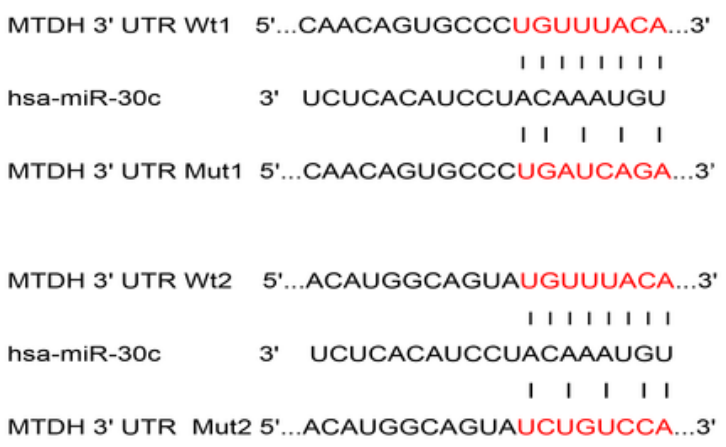

D

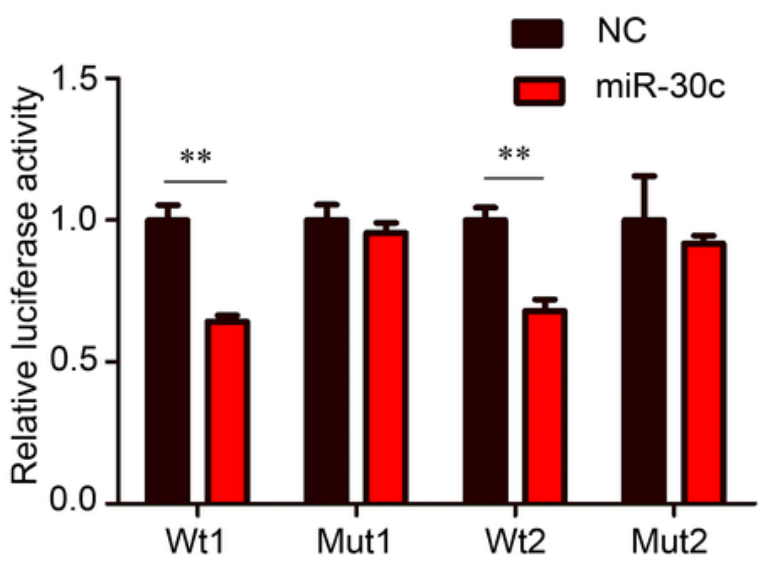

$\mathrm{F}$

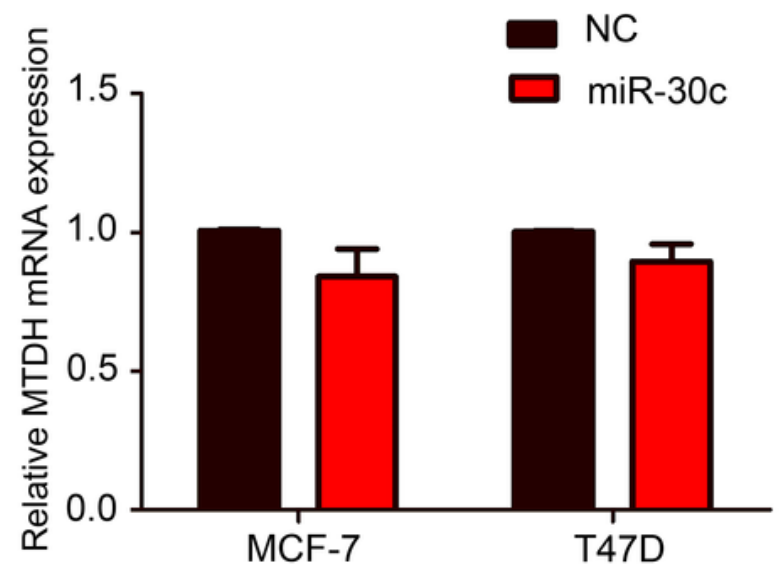

\section{Figure 1}

MiR-30c inhibits MTDH expression by directly targeting its 3'UTR.a MiR-30c target gene prediction by TargetScan, PicTar and DIANA algorithms. The overlapping genes are MTDH, LHX8, and EED. b Two predicted miR-30c target sites reside at nucleotides 287-294 and 1569-1576 of the MTDH 3'UTR; Wt: 
wild type, Mut: mutant. c The alignment of miR-30c target sequences in the MTDH 3'UTR from five mammals. The evolutionarily conserved nucleotides are highlighted in red. $d$ Relative luciferase activity was analyzed after the Wt or Mut reporter plasmids were cotransfected into HEK293 cells with miR-30c mimics or negative control. e Western blot analysis of MTDH protein levels in MCF-7, T47D and MDA-MB231 cell lines transfected with miR-30c mimics $(20 \mu \mathrm{M}, 5 \mu \mathrm{l})$ or negative control. f Relative MTDH mRNA levels were determined by quantitative real-time PCR (qPCR). GAPDH was used as the endogenous control for MTDH mRNA level analysis. Columns, average of at least three biological repeats; bars, s.d. ${ }^{*} \mathrm{p}<0.05 ;{ }^{* *} \mathrm{p}<0.01$. 
A

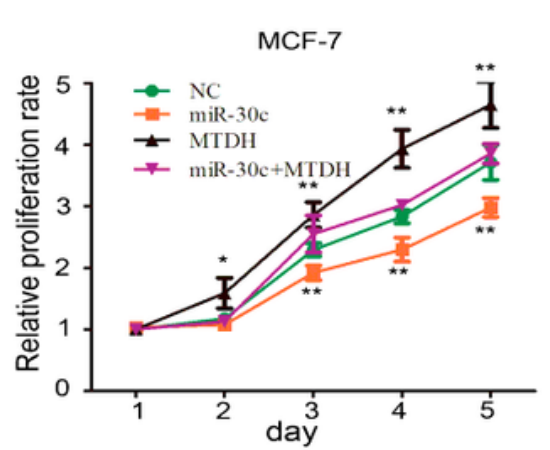

D

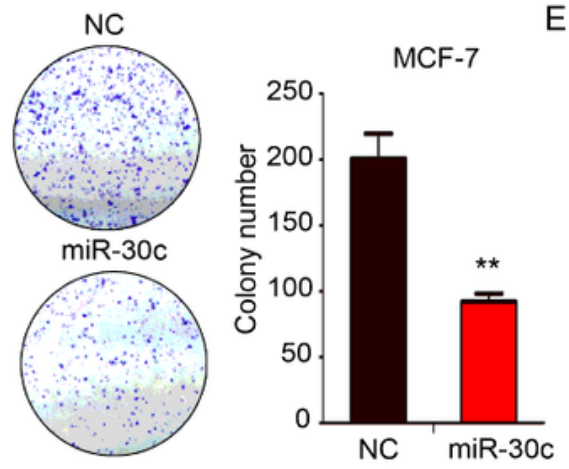

B

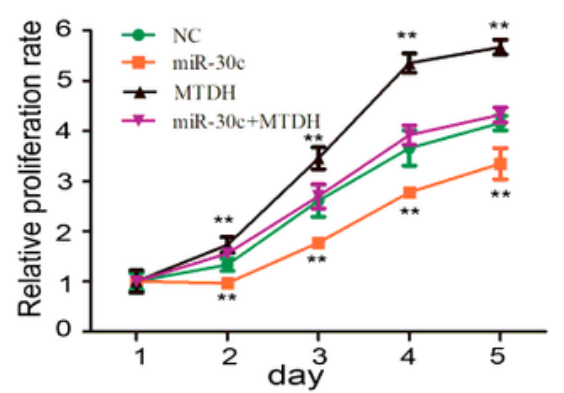

E
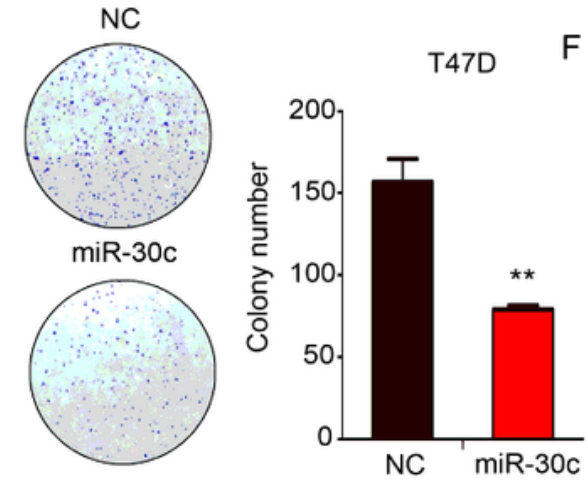

C

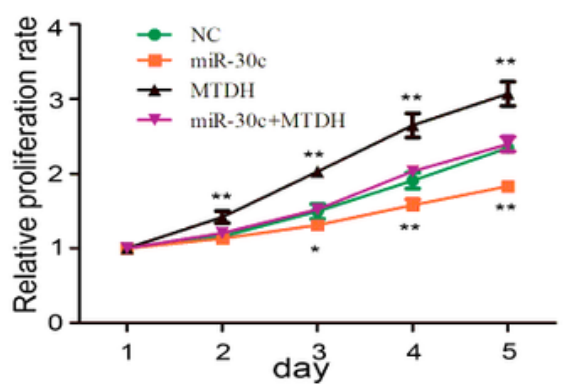

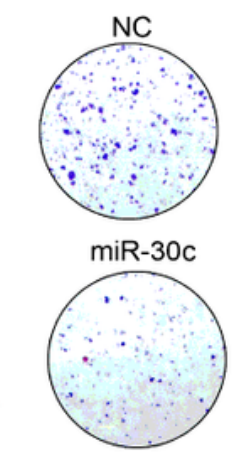

BT474

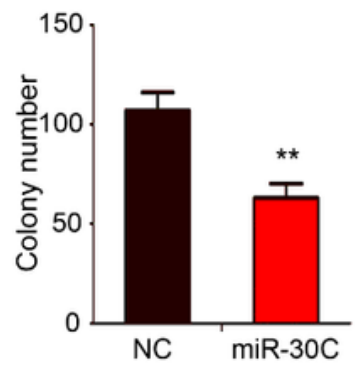

K

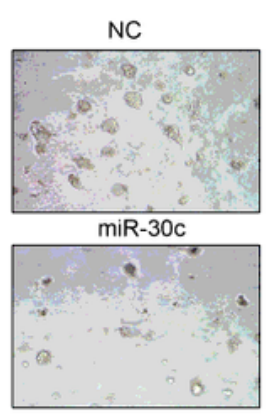

$\mathrm{J}$

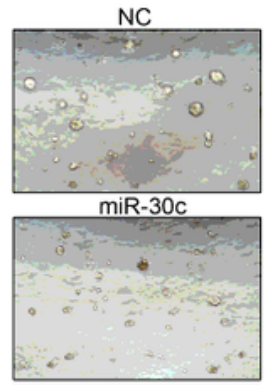

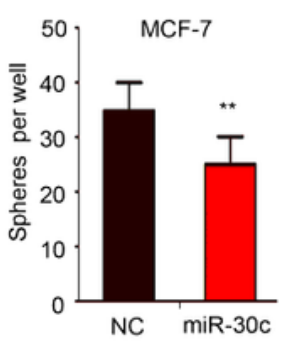

$\mathrm{L}$

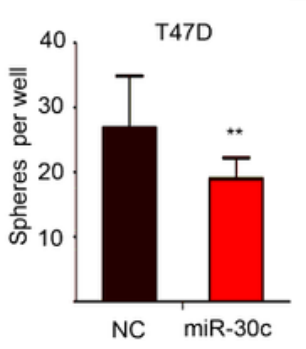

pLKO.1
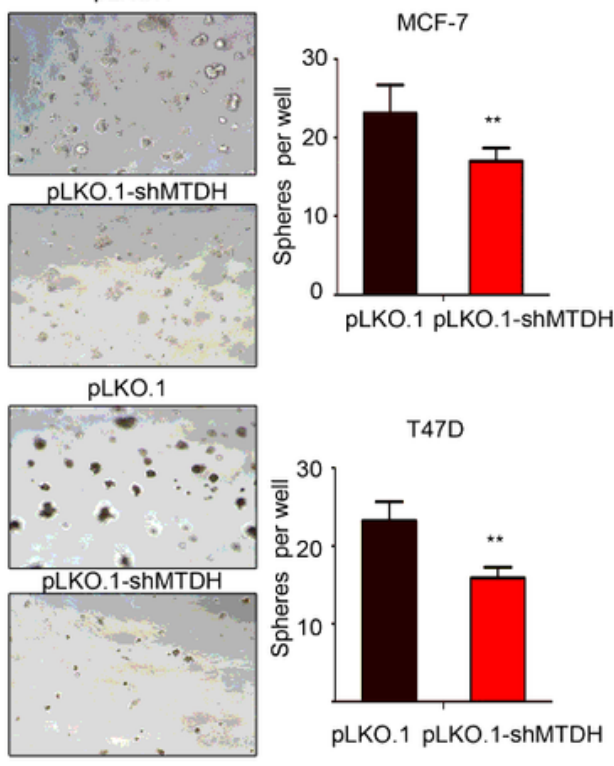

\section{Figure 2}

MiR-30c inhibits breast cancer cell proliferation in vitro by targeting MTDH. a, b and c Growth curves are presented after MCF-7, T47D and BT474 cells were transfected with miR-30c mimics or negative control together with either LV6-MTDH or LV6-vector. Cell growth was quantified and normalized to that at day 0. $d$, e and f Colony growth assays were performed on MCF-7, T47D and BT474 cells with miR-30c mimics or negative control. The number of colonies was counted and compared in the diagrams. $\mathrm{g}$, h Western 
blotting assay of MTDH in MCF-7 and T47D cells transfected with miR-30c mimics or negative control. $\beta$ Actin was used as the endogenous control. i, j Tumor sphere formation assays were performed on MCF-7 and T47D cells transfected with miR-30c mimics or negative control. The number of colonies was counted and compared in the diagrams. $k$, I Tumor sphere formation assays were performed on MCF-7 and T47D cells transfected with pLK0.1-shMTDH or pLKO.1. The number of colonies was counted and compared in the diagrams. Columns, average of at least three biological repeats; bars, s.d. ${ }^{*} p<0.05$; $\star \star x<0.01$.
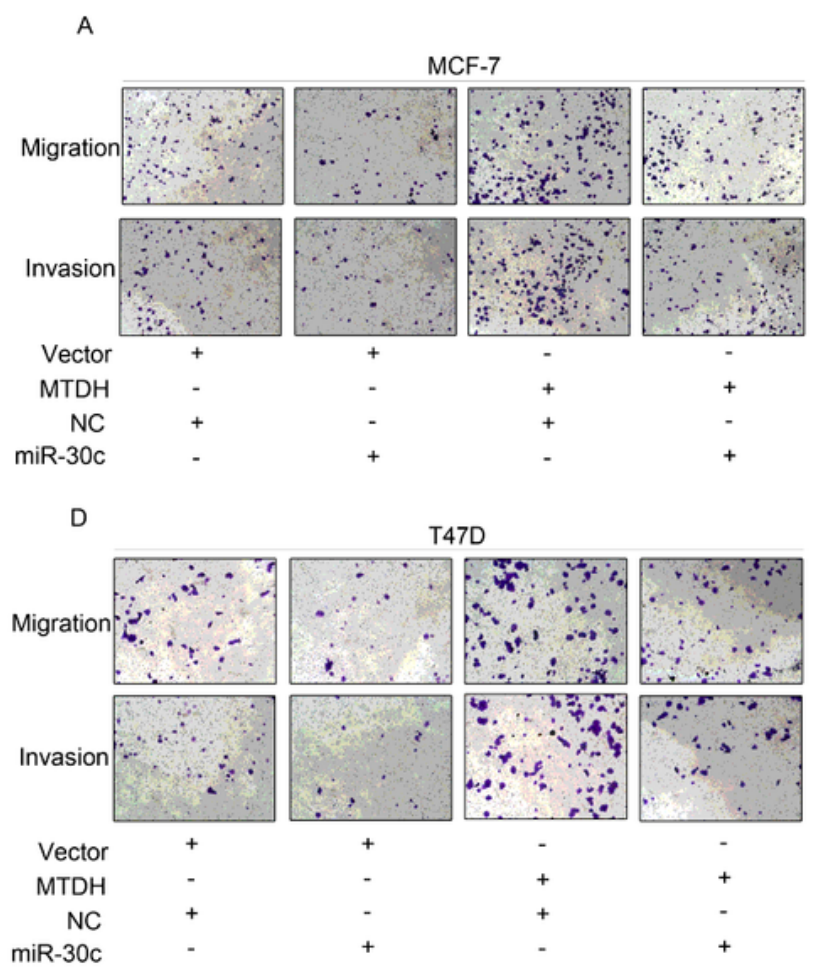

G

BT474
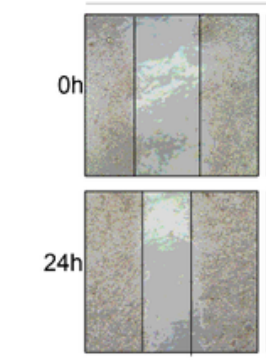

Vector $\mathrm{MTDH}$

$\mathrm{NC}$
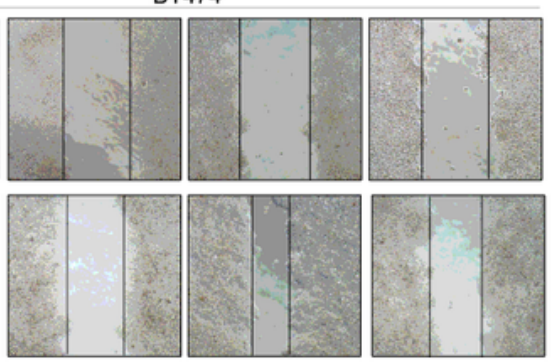

$\operatorname{miR}-30 \mathrm{c}$

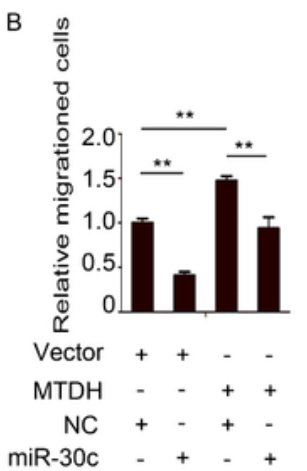

E

B

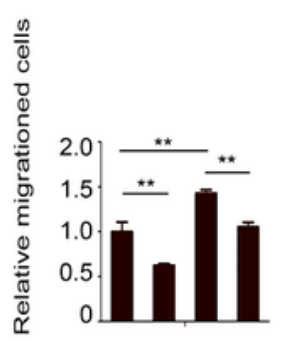

Vector + + - -

$\begin{array}{llll}\mathrm{MTDH} & - & - & + \\ \mathrm{NC} & + & - & +\end{array}$

$\mathrm{NC}$

$\mathrm{H}$

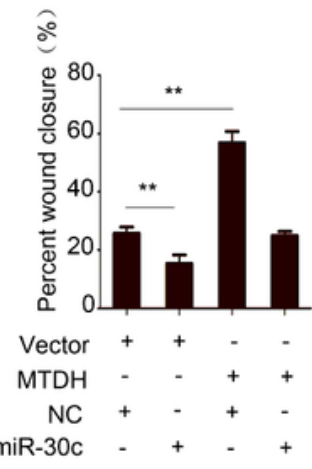

C

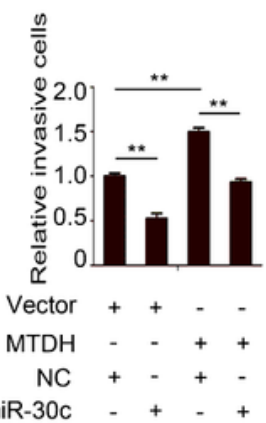

$\mathrm{F}$

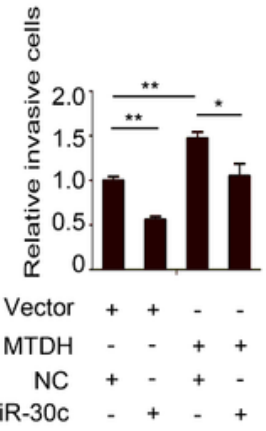




\section{Figure 3}

MiR-30c inhibits breast cancer cell migration and invasion in vitro by targeting MTDH. a, $d$ Transwell assays were performed to examine the migration and invasion abilities of MCF-7 and T47D cells transfected with miR-30c mimics or negative control together with either LV6-MTDH or LV6-vector. b, c and $e, f$ The relative migration and invasion abilities of MCF-7 and T47D cells were analyzed quantitatively. g, h Wound healing of BT474 cells transfected with miR-30c mimics, LV6-MTDH or control vectors. The percentage of wound closure is presented and compared in the diagrams. Columns, average of at least three biological repeats; bars represent s.d.; ${ }^{*} p<0.05 ; * * p<0.01$. 


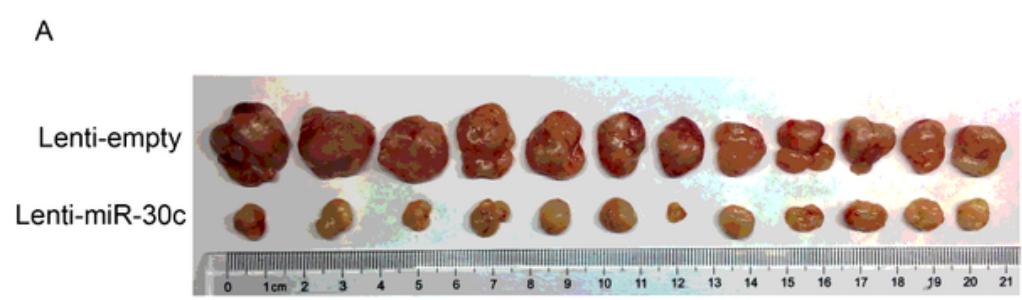

C

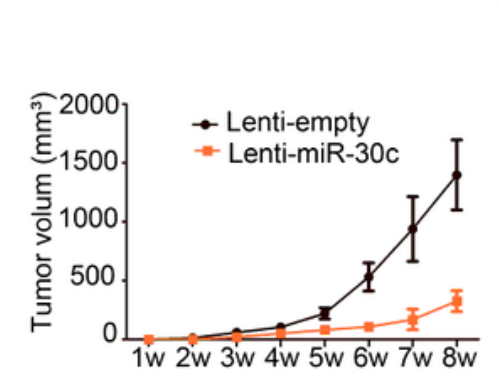

D

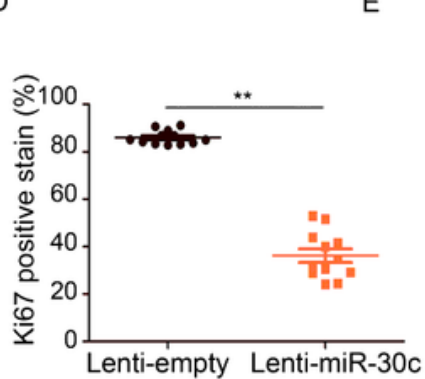

$\mathrm{F}$

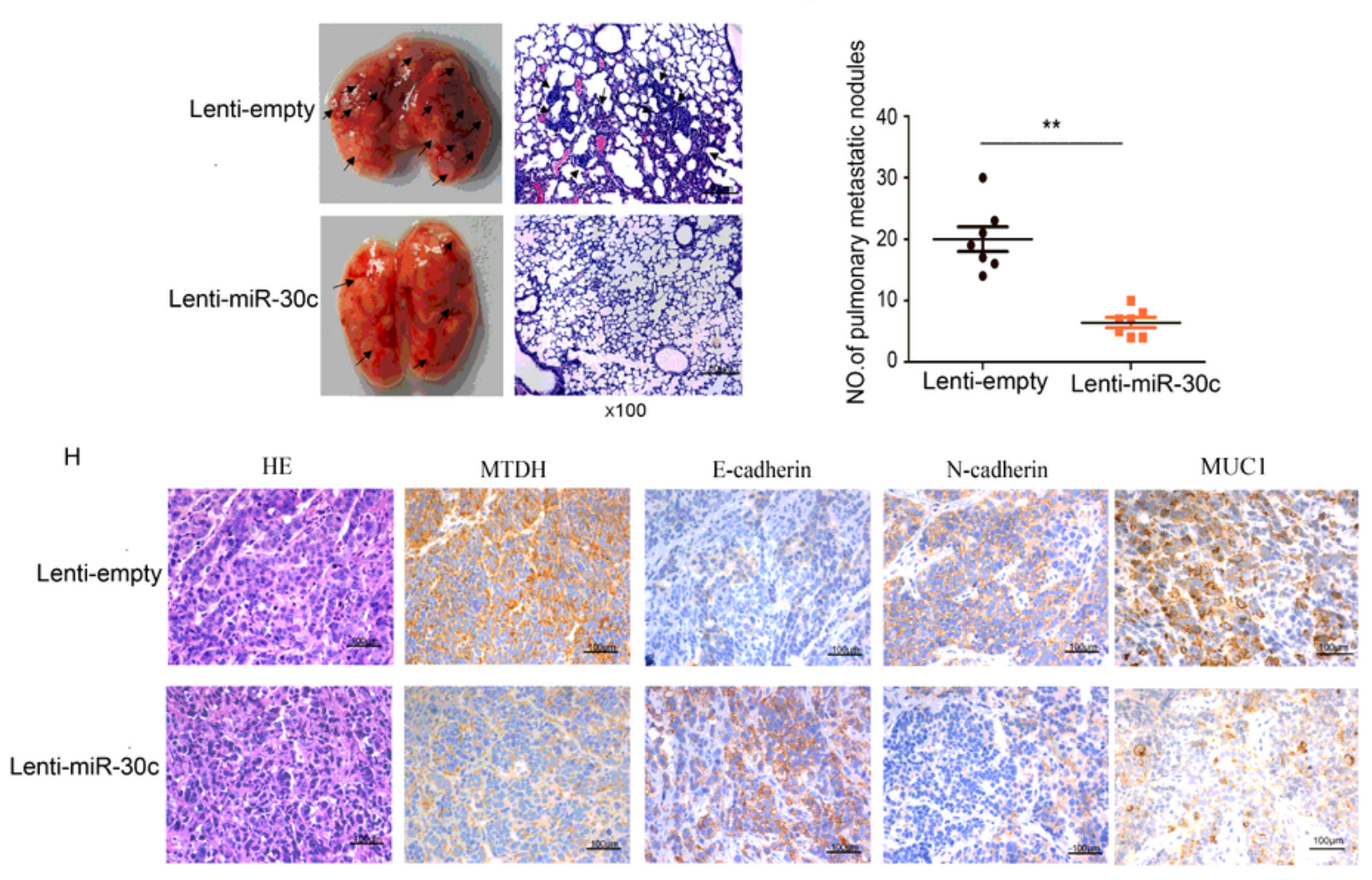

B
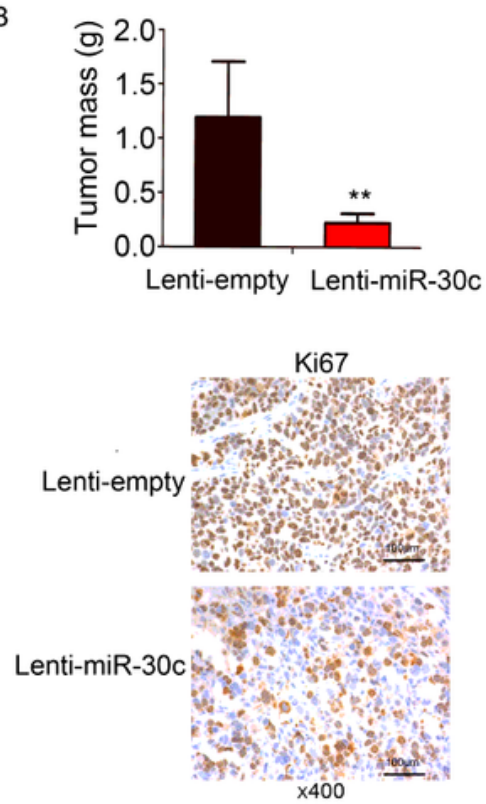

\section{Figure 4}

miR-30c inhibits tumorigenesis and metastasis in vivo. a MCF-7 cells transfected with lenti-miR-30c or lenti-empty were inoculated subcutaneously into the flanks of BALB/c female mice $(n=12)$, as described in the Materials and Methods. b Sixty days after injection, the mice were sacrificed, necropsies were performed, and the tumors were weighed. c The size of tumors in the two groups was measured and calculated and compared every week. d, e Histological examination of the tumor xenografts. 
Immunohistochemical analysis revealed Ki67 expression in xenografts (magnification at 400x). $\mathrm{f} \mathrm{MDA-}$ MB-231 cells with lenti-miR-30c or lenti-empty were injected into the tail veins of nude mice $(n=7$ per group). Four weeks after injection, mice were sacrificed. The numbers of pulmonary metastatic nodules in the lung were counted and compared with Student's t-test. Representative images of lung metastasis in each group (left). The micrometastases of the two groups in the lung per H\&E stained section from individual mice were present (right). $g$ The numbers of pulmonary metastatic nodules in each group were calculated and compared. h H\&E and IHC analysis shows the expression of MTDH, E-cadherin, N-cadherin and MUC1 in xenografts (magnification at 400x).Columns, average of at least three biological repeats; bars represent s.d.; ${ }^{*} p<0.05 ;{ }^{*} \mathrm{p}<0.01$. 

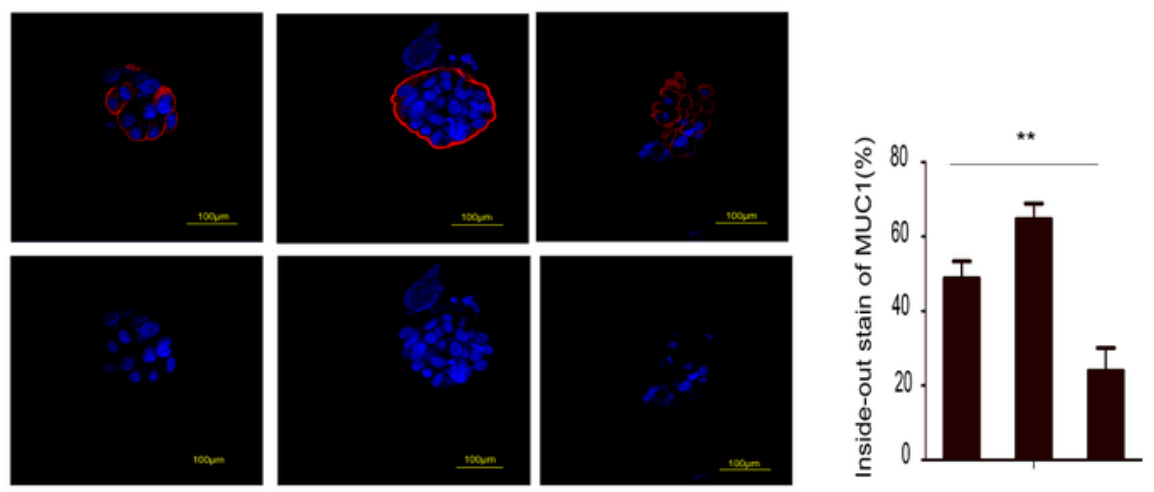

Nucleus/MUC1

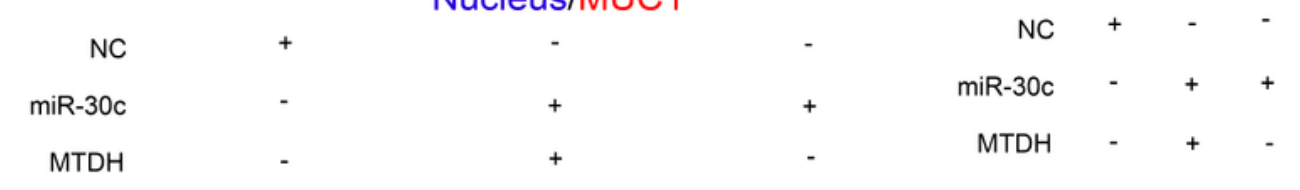

C

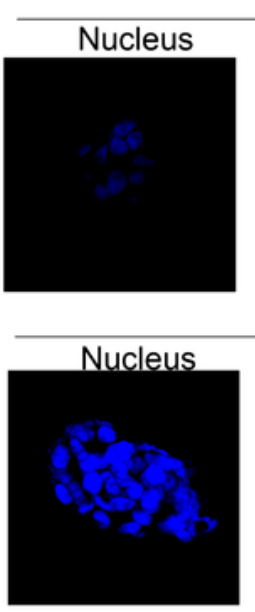

LV6-vector

D

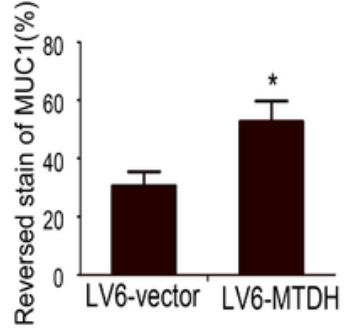

E

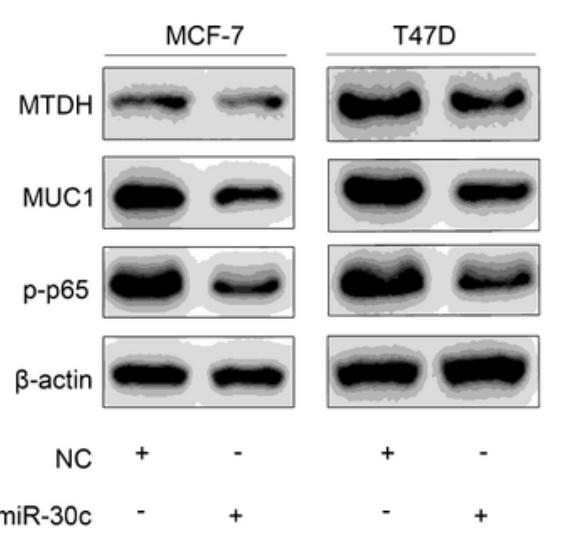

F

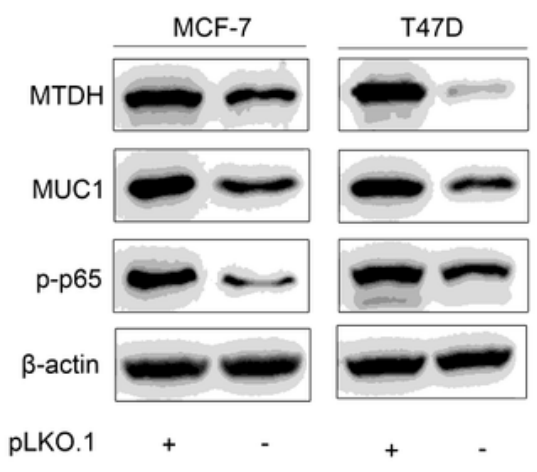

pLKO.1-shMTDH

\section{Figure 5}

miR-30c negatively regulates cell polarity by targeting MTDH. a, c Immunofluorescence image of the inside-out staining pattern of MUC1 in IMPC primary cells transfected with miR-30c mimics, LV6-MTDH or LV6-vector. b, $d$ The inside-out staining pattern of MUC1 was analyzed quantitatively in IMPC primary cells transfected with miR-30c mimics, LV6-MTDH or LV6-vector. e MUC1 and p-p65 protein levels were analyzed after MCF-7 and T47D cells were transfected with miR-30c mimics or negative control. f MUC1 
and p-p65 protein levels are presented after MCF-7 and T47D cells were transfected with pLKO.1-shMTDH or pLKO.1. Columns, average of at least three biological repeats; bars represent s.d.; ${ }^{*} p<0.05$; ${ }^{*} p<0.01$.

A

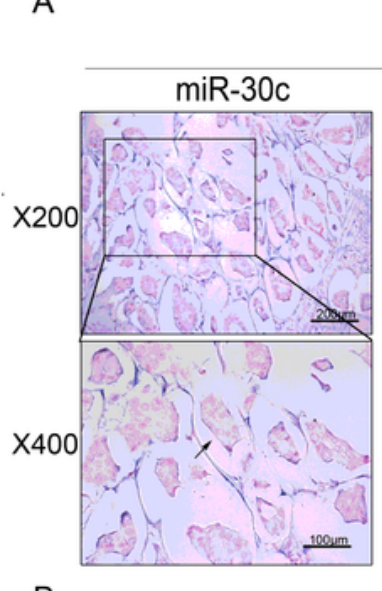

B

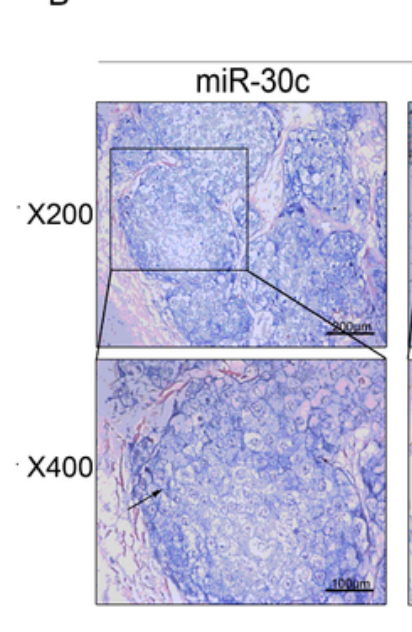

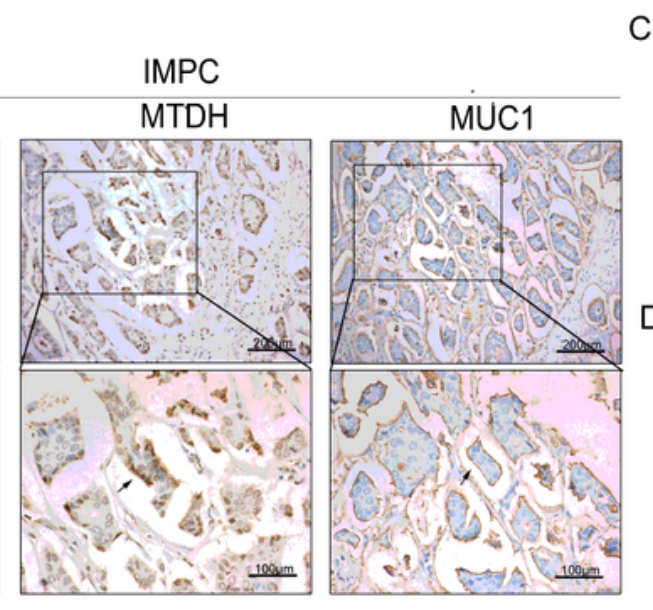

IDC-NST
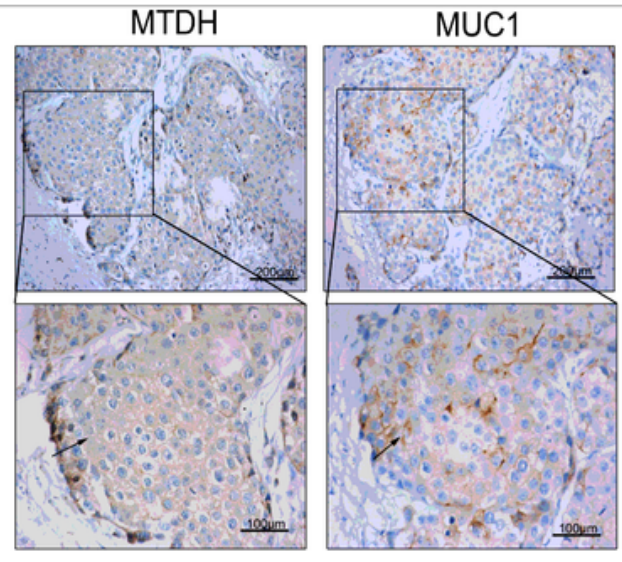

C

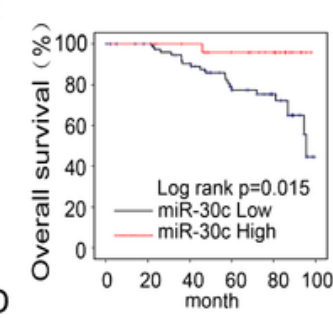

D

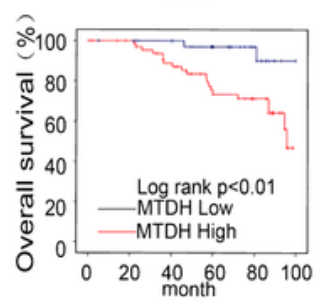

E

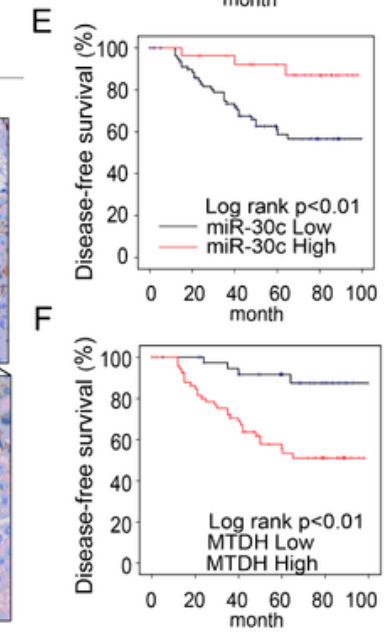

G

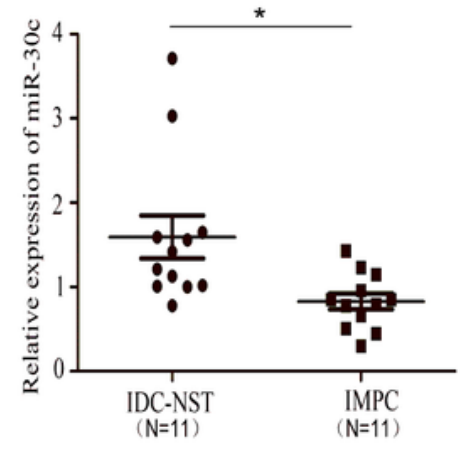

$\mathrm{H}$

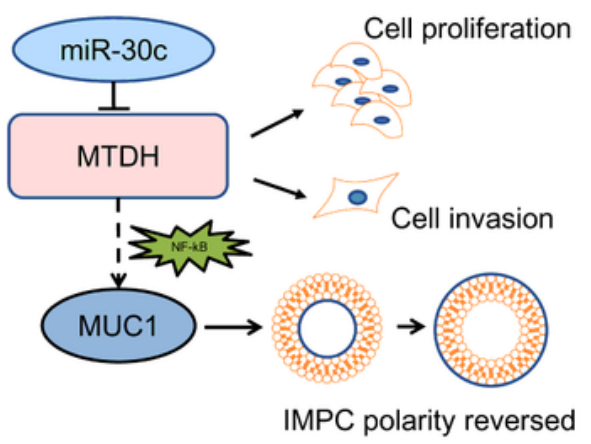

\section{Figure 6}

MiR-30c negatively regulated MTDH expression and correlated with the prognosis of IMPC.The miR-30c expression levels were analyzed in IMPC and IDC-NST tissues by in situ hybridization (ISH). MTDH and MUC1 expression levels were analyzed in IMPC and IDC-NST tissues by immunohistochemistry (IHC). a, b 
Representative images of miR-30c, MTDH and MUC1 expression in IMPC and IDC-NST cells. c, e The overall survival and disease-free survival of patients with high or low miR-30c expression in IMPC patients. d,f The overall survival and disease-free survival of patients with high or low MTDH expression in IMPC patients. g QPCR analysis shows the expression levels of miR-30c in 11 pairs of tissues. Expression for the gene was normalized to U6 expression. ${ }^{*} \mathrm{p}<0.05$. $\mathrm{h}$ A schematic showing how the signaling pathway of miR-30c regulates tumor cell invasion, metastasis and polarity reversal in invasive micropapillary carcinoma of the breast.

\section{Supplementary Files}

This is a list of supplementary files associated with this preprint. Click to download.

- Supplementarytable5.docx

- Supplementarytable4.docx

- Supplementarytable3.docx

- Supplementarytable2.docx

- Supplementarytable1.docx

- Supplementaryfig3.tif

- Supplementaryfig2.tif

- Supplementaryfig1.tif 